\title{
Supramolecular Structure of Polypeptides in Concentrated Solutions and Films. I. Origin of Rod-like Scattering and Effect of Form-Optical Rotation
}

\author{
Takeji Hashimoto, Toshikazu IJITsu,* Kiyonaga YAmAguCHI,** \\ and Hiromichi KAWAI \\ Department of Polymer Chemistry, Faculty of Engineering, Kyoto University, \\ Yoshida, Sakyo-ku, Kyoto 606, Japan.
}

(Received April 10, 1980)

\begin{abstract}
Supramolecular structures of poly( $\gamma$-benzyl L-gultamate) in concentrated solutions of helicogenic solvents and in solid state were investigated by laser-light scattering technique. The depolarized elastic scattering indicates that there exists two types of rod-like scattering with $\mathrm{X}$-type (having maximum intensity at odd multiples of the azimuthal angle, $\mu=45^{\circ}$ ) and +-type (having maximum intensity at multiples of $\mu=90^{\circ}$ ) angular distributions. The structural origin of such rod-like scattering and the effect of form-optical rotation on the rod-like scattering are discussed.
\end{abstract}

KEY WORDS Supramolecular Structure / Poly( $\gamma$-benzyl L-gultamate) / Liquid Crystals / Depolarized Light Scattering / Form Optical Rotation / Rod-like Scattering /

Laser-light scattering has been used as a technique for characterizing the supramolecular structure of polymeric systems in solutions and in the solid state in terms of their size, shape, and internal molecular orientation. ${ }^{1-3}$ In this paper we shall investigate the light scattering, especially, the depolarized components of the scattered light from the supramolecular structure of poly $(\gamma$-benzyl Lgultamate) (PBLG) occurring in concentrated solutions of helicogenic solvents and in solid films obtained by casting these solutions.

\section{TEST SPECIMENS}

PBLG specimens were prepared by polymerizing $N$-carboxyanhydride of $\gamma$-benzyl L-glutamate in a mixture of dioxane-dichloromethane $(1: 1, \mathrm{v} / \mathrm{v})$ with triethylamine as an initiator. The polymers were further fractionated. The polymers have a

* Present address: The Central Research Laboratory, Idemitsu Kosan Co., Ltd., Sodegaura-cho, Kimitsu-gun, Chiba 299-02, Japan.

** Present address: Materials $R \& D$ Division, The Yokohama Rubber Co., Ltd., Hiratsuka, Kanagawa 254, Japan. heterogeneity index $\left(M_{w} / M_{n}\right)$ approximately equal to 1.3 from gel-permeation chromatography (GPC) measured on a dimethylformamide solution. From viscosity measurements in dichloroacetic acid at $25^{\circ} \mathrm{C}$, the PBLG specimens used in this work have molecular weights of $1.56 \times 10^{5}$ (designated as PBLG-1) and $1.06 \times 10^{5}$ (designated as PBLG-2). The difference in the molecular weights of the two specimens is of no significance in this study.

The PBLG films were prepared by casting $0.1-$ $10 \mathrm{wt} \%$ solutions of helicogenic solvents, such as chloroform, dioxane, tetrachloroethane (TCE), benzene, ethylene dichloride, $m$-cresol, and $N, N^{\prime}$ dimethylformamide (DMF) onto a glass plate. Light-scattering studies were also conducted during the solvent evaporation process or in the equilibrium state (attained by enclosing the solutions into a cell), as a function of concentration.

\section{LIGHT-SCATTERING INTENSITY DISTRIBUTIONS}

\section{Scattering from PBLG Films}

Figure 1 shows typical depolarized lightscattering patterns observed for PBLG-1 films cast 


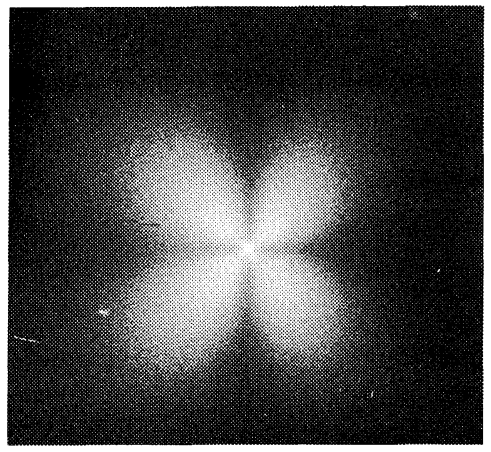

(a) PBLG-DMF

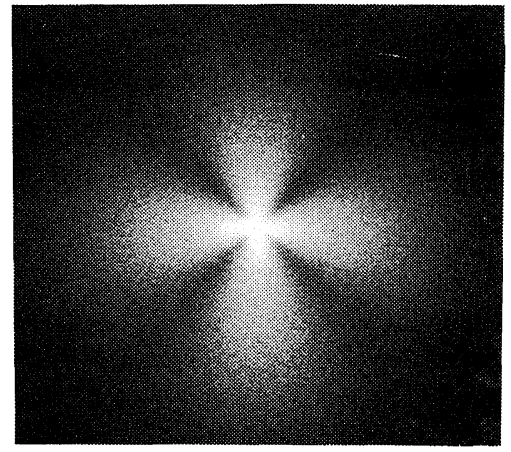

(b) PBLG-TCE

Figure 1. Typical $H_{\mathrm{v}}$ light-scattering patterns from PBLG-1 films cast from $5 \mathrm{wt} \% \mathrm{DMF}$ solution (a) and $1 \mathrm{wt} \%$ TCE solution (b). Corresponding supramolecular structures are shown in Figures 5, 7 and 8.

from $5 \mathrm{wt} \%$ DMF solutions (a) and $1 \mathrm{wt} \%$ TCE solutions (b). The patterns were obtained with a vertically polarized incident beam and a horizontally polarized analyzer (i.e., $H_{\mathrm{v}}$-polarization). Patterns (a) and (b) have a four-fold symmetry with respect to the azimuthal angle $(\mu)$ dependence of the scattered-light intensity. The intensity continuously drops with an increase in the scattering angle $\theta$. The radial distribution of the scattered intensity is typical of so-called rod-like scattering. ${ }^{4}$

There are two types of the rod-like scattering: (i) one has an X-type $\mu$-dependence with the intensity maxima at odd multiples of $\mu=45^{\circ}$ (Figure 1(a)), and (ii) the other has +-type $\mu$-dependence with the intensity maxima at multiples of $\mu=90^{\circ}$ (Figure 1(b)). Only DMF gave films showing the X-type pattern among the casting solvents used in this work.

Such rod-like scattering patterns have been interpreted in terms of a random assembly of optically anisotropic rod model in a twodimensional plane $e^{4-8}$ normal to the propagating direction of incident beam or in three-dimensional space. $^{6,8-11}$ Figure 2 shows these two types of rod models. The rods are assumed to be composed of uniaxially anisotropic scattering elements with polarizabilities $\alpha_{\|}$and $\alpha_{\perp}$ parallel and perpendicular to the optical axes, respectively.

In the rod of model A, the optical axis designated as a unit vector $\boldsymbol{d}$ is confined to a particular plane and makes polar angle $\omega_{0}$ with respect to the rod axis designated as a unit vector $\boldsymbol{r}_{1}$ (see Figure 2(a)). The rod as a whole may be randomly rotated with

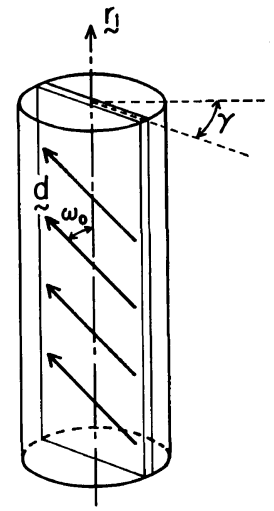

(a)

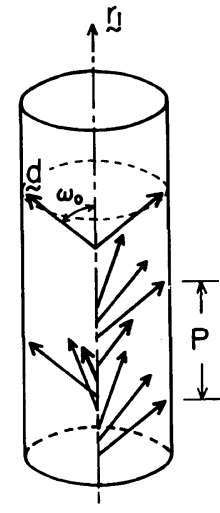

(b)
Figure 2. Two types of anisotropic rods: (a) in the rod of model A, the optical axis $\boldsymbol{d}$ is confined to a particular plane defined by the angle $\gamma$ and makes a polar angle $\omega_{0}$ with respect to the rod axis $\boldsymbol{r}_{1}$ and (b) in the rod of model B $\boldsymbol{d}$ is oriented in cylindrically symmetry with $\boldsymbol{r}_{1}$, i.e., $\omega_{0}$ being fixed but $\gamma$ varying randomly within the rod.

respect to the angle $\gamma$ in a random orientation. As a supplementary system to the rod of model A, the rod of model B has a cylindrically symmetric optical axis orientation within the rod, i.e., having a fixed value for the polar angle $\omega_{0}$ and randomly varying $\gamma$ within the rod (see Figure 2(b)).

It has been reported by Hashimoto et al. ${ }^{11}$ that the rod of model A gives rise to the X-type $H_{\mathrm{V}}$ patterns for $\omega_{0}$ satisfying $0^{\circ} \leqq \omega_{0}<\left(\omega_{01}\right)_{c}$ and $\left(\omega_{02}\right)_{\mathbf{c}}<\omega_{0} \leqq 90^{\circ}$ and +-type patterns for $\omega_{0}$ satisfying $\left(\omega_{01}\right)_{\mathrm{c}}<\omega<\left(\omega_{02}\right)_{\mathrm{c}} ; \quad\left(\omega_{01}\right)_{\mathrm{c}}=30^{\circ} 33^{\prime} \quad$ and $\left(\omega_{02}\right)_{\mathrm{c}}=70^{\circ} 07^{\prime}$ for the random assembly of the rod 


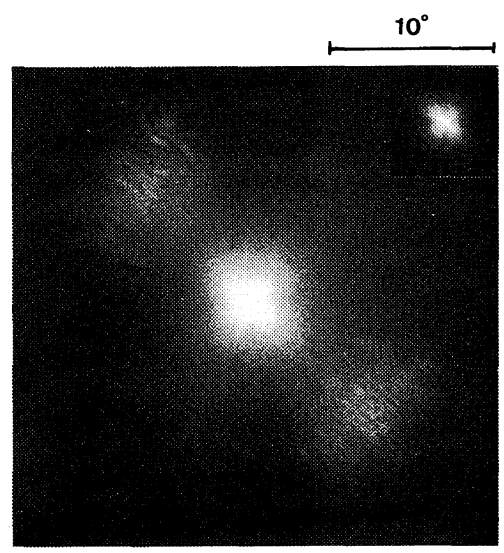

Figure 3. $H_{\mathrm{v}}$ pattern obtained during solvent evaporation process of $1 \mathrm{wt} \%$ TCE solution of PBLG-2. The pattern at the upper right corner of the figure shows a small angle scattering pattern taken with an appropriate exposure time and which corresponds to the overexposed pattern at the center of the pattern.

in three-dimensional space or $\left(\omega_{01}\right)_{\mathrm{c}}=22^{\circ} 30^{\prime}$ and $\left(\omega_{02}\right)_{\mathrm{c}}=67^{\circ} 30^{\prime}$ for the random assembly of rods in two-dimensional space. ${ }^{9}$ It may be noted that $P_{4}\left(\cos \omega_{0}\right)=0$ for $\omega_{0}=30^{\circ} 33^{\prime}$ and $70^{\circ} 07^{\prime}\left(P_{4}(x)\right.$ being the 4-th order Legendre function of the first kind) and $\cos 4 \omega_{0}=0$ for $\omega_{0}=22^{\circ} 30^{\prime}$ and $67^{\circ} 30^{\prime}$. On the other hand the rod of model $\mathrm{B}$ gives rise to $\mathrm{X}$-type $H_{\mathrm{v}}$ patterns regardless of the value of $\omega_{0},{ }^{11}$ which only affects the absolute intensity level according to $\left[P_{2}\left(\cos \omega_{0}\right)\right]^{2}$

Consequently the two types of $H_{\mathrm{v}}$ patterns shown in Figure 1 reflect a difference in the molecular orientation within the rod-like structure. The Xtype pattern is ascribed to the rod of model A having the value of $\omega_{0}$ satisfying $0^{\circ} \leqq \omega_{0}<\left(\omega_{01}\right)_{\mathbf{c}}$ or $\left(\omega_{02}\right)_{\mathrm{c}}<\omega_{0} \leqq 90^{\circ}$ or to the rod of model $\mathrm{B}$. The +type pattern is ascribed to the rod of model A having the value of $\omega_{0}$ satisfying $\left(\omega_{01}\right)_{\mathbf{c}}<$ $\omega_{0}<\left(\omega_{02}\right)_{c}$. In the next section (section of the STRUCTURAL ENTITIES OF THE ROD MODELS) we shall clarify the structural entity of such rod models.

\section{Scattering from PBLG Concentrated Solutions}

Figure 3 shows an $H_{\mathrm{v}}$ pattern obtained during the solvent evaporation process of $1 \mathrm{wt} \%$ TCE solution of PBLG-2. As will be discussed in the next section, this pattern arises from the cholesteric liquidcrystalline texture formed in concentrated solutions $\mathrm{s}^{12-14}$ during the solvent-evaporation process.
The scattering maximum which appears at around $\theta \simeq 10^{\circ}$ corresponds to one half of the cholesteric pitch $P^{15}$, the scattering angle of the maximum intensity in the medium $\theta_{\mathrm{m}}^{*}$ and a distance $S=P / 2$ being interrelated by Bragg's equation,

$$
2 S \sin \left(\theta_{\mathrm{m}}^{*} / 2\right)=\lambda_{\mathrm{m}}
$$

where $\lambda_{\mathrm{m}}$ is the wavelength of light in the medium.

The scattering from the cholesteric pitch is asymmetric with $\mu$-dependence, the intensities at $\mu=135$ and $315^{\circ}$ being much stronger than those at $\mu=45$ and $225^{\circ}$. This asymmetry is primarily attributed to the effect of the form-optical rotation on the scattering pattern whose details will be discussed elsewhere. ${ }^{15,16}$ In this paper, we shall focuss our attentions on the small-angle scattering appearing in the center of the pattern. Since the small-angle scattering is overexposed, we showed the corresponding pattern taken with an appropriate exposure time at the upper right corner of the figure.

The small-angle scattering has the characteristic feature of rod-like scattering and can be attributed to the rod of model B with $\omega_{0}=90^{\circ}$ as will be discussed in next section. The asymmetry of its $\mu$ dependence is again observed in that the intensities at $\mu=135$ and $315^{\circ}$ are stronger than those at $\mu=45$ and $225^{\circ}$. This is due to the form-optical rotation on the rod-like scattering to be discussed in detail in section of EFFECT OF FORM-OPTICAL ROTATION ON THE ROD-LIKE SCATTERING. These aspects of scattering as observed in a nonequilibrium state can also be observed in an equilibrium state.

Figure 4 shows the scattering patterns from a concentrated solution in equilibrium state $(17 \mathrm{wt} \%$ dioxane solution of PBLG-2 in a glass cell of thickness $0.15 \mathrm{~mm}$ ). Again the small angle scattering patterns have the characteristic feature of rod-like scattering attributed to the rod of model $B$ with $\omega_{0}=90^{\circ}$ and the wide-angle scattering maximum is again attributed to the uniformity of the cholesteric pitch. The asymmetry of the pattern is due to the effect of the form-optical rotation.

\section{STRUCTURAL ENTITIES OF THE ROD MODELS}

\section{Supramolecular Structure in Solid State}

Figure 5 shows electron micrographs (two-stage replica) of the PBLG-1 film cast from a DMF 
PBLG-Dioxane $16.9 \mathrm{wt} \%$

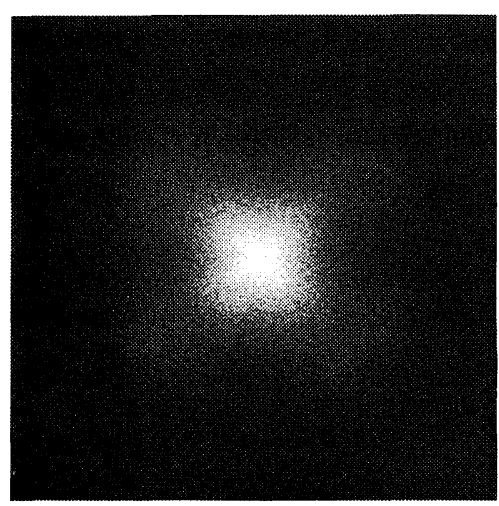

$H_{\mathbf{v}}$

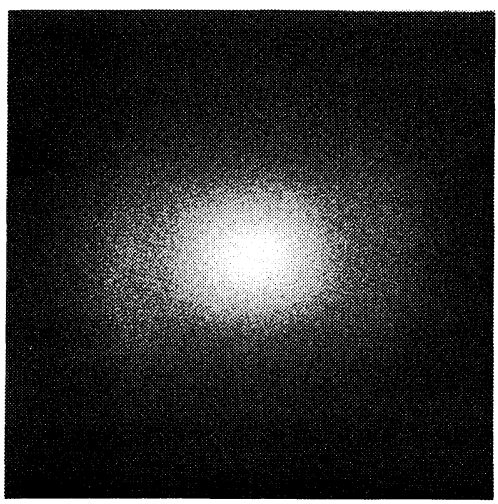

$V_{\mathbf{v}}$

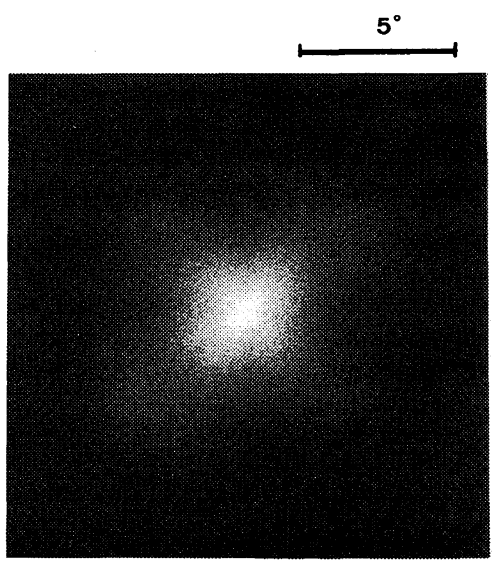

$V_{\mathrm{H}}$

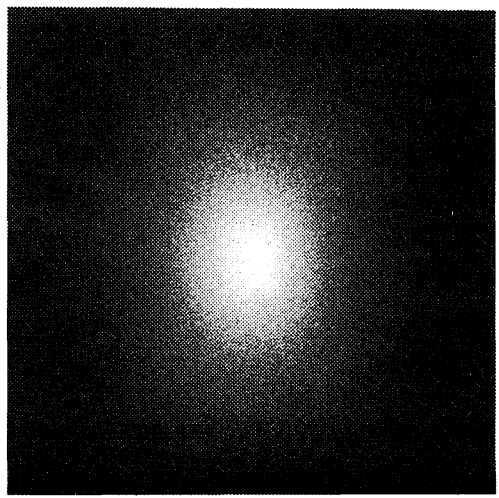

$H_{\mathrm{H}}$

Figure 4. Depolarized and polarized components of scattering from a concentrated solution in a glass cell. A $17 \mathrm{wt} \%$ dioxane solution of PBLG-2.

solution, giving rise to the scattering pattern shown in Figure 1(a). The micrograph at low magnification clearly indicates a rod-like supramolecular structure oriented randomly in space (Figure 5(a)). The micrograph at a higher magnification exhibits fine striations in the rod-like texture, indicating that the rod corresponds to the superhelix (or coiled coil) in which the $\alpha$-helices are packed together in a multistranded rope..$^{17}$ The size and shape of the rod depend on the conditions of the film preparation.

Owing to the coiled coil structure, the optical axis $\boldsymbol{d}$ rotates around the rod axis $\boldsymbol{r}_{1}$ with a pitch much smaller than the length of the rod as a whole. The small-angle depolarized light scattering shown in Figure 1(a) reflects the orientation correlation of the optical axis occuring at a large correlation distance, i.e., the distance which is greater than the pitch of the coiled coil structure. Consequently the optical axis $\boldsymbol{d}$ is regarded to rotate randomly around $\boldsymbol{r}_{1}$, as in the model $\mathrm{B}$, for the small-angle scattering. The relative scattered intensity distribution depends on the size and shape of the rod-like structure, while the absolute intensity primarily depends on $\left[\left(\alpha_{\|}-\alpha_{\perp}\right)\right.$ $\left.P_{2}\left(\cos \omega_{0}\right)\right]^{2}$, i.e., anisotropy $\left(\alpha_{\|}-\alpha_{\perp}\right)$ of the scattering volume element and orientation $\omega_{0}$ of the optical axis.

Figures 6 to 8 show the optical and electron micrographs of the film specimens giving rise to the +-type $H_{\mathrm{v}}$ pattern shown in Figure 1(b). Figure 6 shows the polarized-light micrograph corresponding to the same portion of the film specimen which gives rise to the $H_{\mathrm{V}}$ pattern in Figure 1(b) (the 


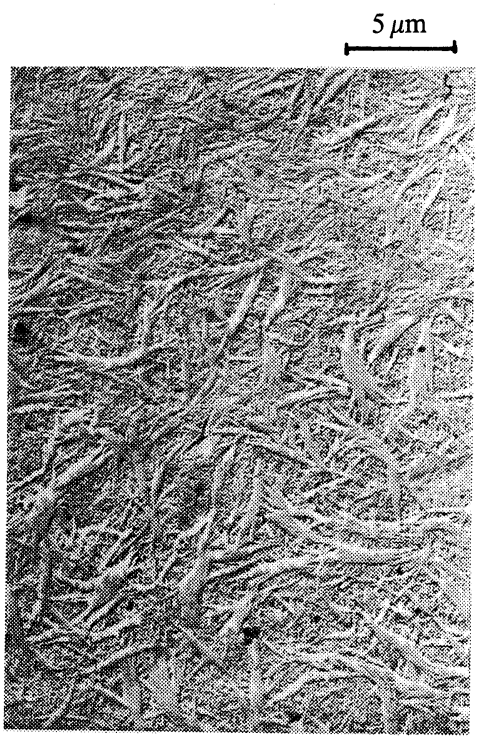

(a)

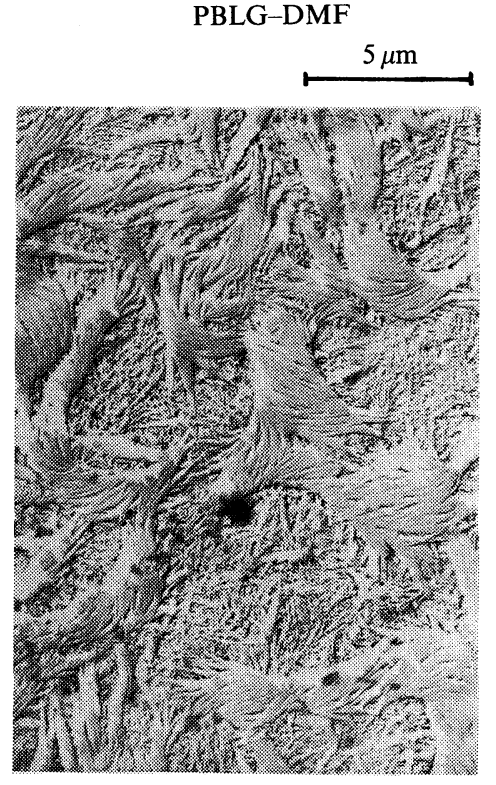

(b)

Figure 5. Electron micrographs (two-stage replica) of the PBLG-1 film cast from a DMF solution. The film gives rise to the scattering pattern shown in Figure 1(a). The micrograph shows right-handed coiled coils (or superhelices).

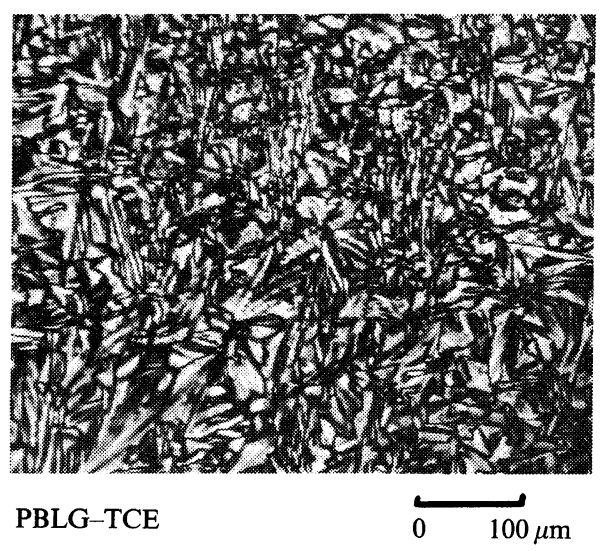

Figure 6. Polarized light micrograph of the same portion of the film specimen giving rise to the $H_{\mathrm{v}}$ pattern in Figure 1(b). Theelectric vectors of the polarizerandanalyzerareset in vertical and horizontal directions.

polarizer and analyzer being set in vertical and horizontal directions). The micrograph indicates a polydomain structure with a strong optical anisotropy.

The fine structure of the domain is not well resolved in this micrograph because of the overlap of the structure along the thickness direction. Therefore the fine structure was studied with a much thinner film specimen prepared by casting a dilute $(0.1 \mathrm{wt} \%$ TCE) solution of PBLG-1. Essentially, the same portion of the same thin-film specimen was investigated by polarized-light microscopy (polarization directions being vertical and horizontal) and depolarized-light scattering (Figure 7) and by transmission electron microscopy (Figure 8). The $H_{\mathrm{v}}$ scattering from the thin film (Figure 7) is essentially identical to that in Figure 1(b), indicating that the domain structure in the thin film should be nearly identical to that in the thicker film.

The fine structure of the domain is clearly resolved in Figure 7 where the domain is shown to be a region having correlated orientation of $\alpha$ helical molecules. The $\alpha$-helices are stacked parallel by end-to-end and side-by-side associations to each other within the domain. The average orientations of the $\alpha$-helices in neighbouring domains are slightly 

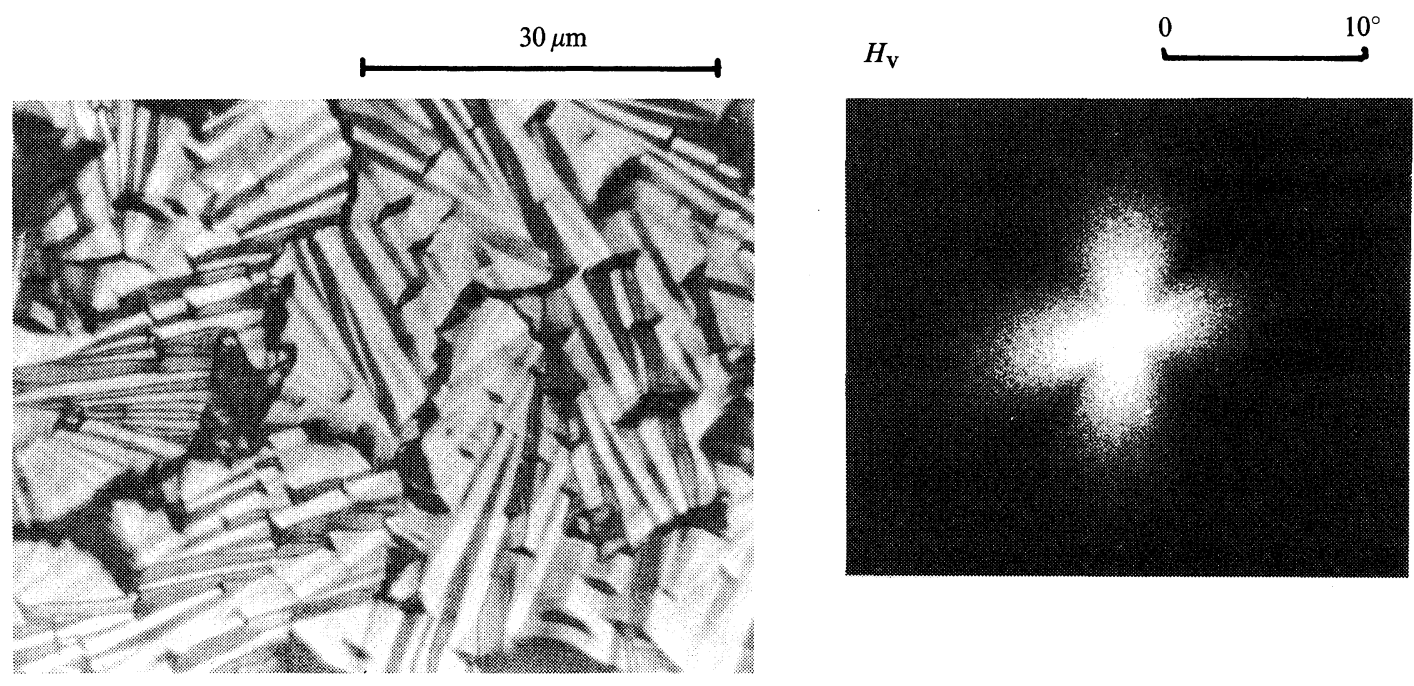

Figure 7. Polarized light micrograph showing a polydomain structure with a strong negative birefringence and corresponding $H_{\mathrm{v}}$ scattering patterns. Thin-film specimens were prepared by casting a dilute solution of PBLG-1 ( $0.1 \mathrm{wt} \%$ TCE). The polarization is set in vartical and horizontal directions.

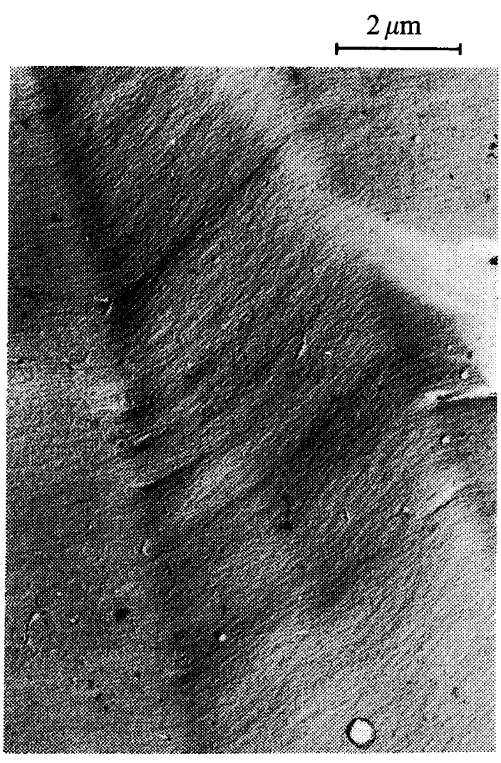

Figure 8. The transmission electron micrograph of the thin film corresponding to Figure 7. Pt-Pd shadowed.

misaligned. The dark domain under the crossed polarizers is attributed to the zero birefringence effect rather than to the zero amplitude effect, and this is confirmed by the fact that the dark domains turn to bright and the bright domains turn to dark when the sample is rotated under crossed polarizers. The domains were found to have negative birefringence from the retardation color.

Figure 8 shows the fine structure of the domain as observed by transmission electron microscopy, the thin film being shadowed by Pt-Pd. If the fine parallel striations observed in each domain are assumed to be parallel to the axes of $\alpha$-helices, the axes make an angle of about $60^{\circ}$ with respect to the longitudinal direction of the domain. Since the optical axis is parallel to the $\alpha$-helical axis, the domain should corrospond to the rod of model A with $\omega_{0} \simeq 60^{\circ}$ which gives rise to the +-type $H_{\mathrm{v}}$ pattern in Figures 1(b) and 7. This assumption may be also justified in consideration of the negative birefringence of the domain, since the $\alpha$-helix of PBLG has a positive birefringence as reported by Robinson, ${ }^{13}$ i.e., the largest refractive index is along the helical axis.

A similar polydomain structure and $H_{\mathrm{V}}$ lightscattering patterns were found for films cast from other solvents, such as chloroform and dioxane. Thus, this domain structure may be a structural entity of the rod of model A. The size and shape of the domain or the correlated region of the optical axis orientation sensitively vary with the conditions of the film preparation. 
Supramolecular Structure in Concentrated Solutions

Figure 9 shows a polarized-light micrograph (a) corresponding approximately to the region giving rise to the scattering pattern shows in Figure 3, which was taken during the solvent evaporation process. The domain or region with alternating bright and dark stripes corresponds to the cholesteric twisted structure with its twisted axis $\boldsymbol{r}_{1}$ nearly in the plane of the paper. The optical axis or the helical axis $\boldsymbol{d}$ is twisted in the domain around $\boldsymbol{r}_{1}$; in a bright region $\boldsymbol{d}$ is perpendicular to $\boldsymbol{r}_{1}$ and in the plane of the paper, and in a dark region, $\boldsymbol{d}$ is perpendicular to the plane of the paper.

The shape of the cholesteric domain is anisotropic and the axes $\boldsymbol{r}_{1}$ are essentially in random orientation. At small-scattering angles where the orientation correlation occurring over a large distance is of importance, scattering arises from the anisotropic domain as a whole. The cholesteric domain should correspond to the structural origin of the rod of model B with $\omega_{0} \simeq 90^{\circ}$ (see Figure 9(b)). Thus, the angular distribution of the small-angle scattering intensity of Figure 3 primarily depends on the size and shape of the cholesteric domain. The internal structure of the rod due to the cholesteric twisted structure does not significantly affect the smallangle scattering, but makes a significant contribution to the scattering at large angles. For example the scattering maximum in the large-angle region occurs at the scattering angle reciprocally related to the identity period $S$ of the alternating bright and white stripes in the micrograph shown in Figure 9(a), i.e., half the distance of the cholesteric pitch. ${ }^{15,16}$

Figure 10 shows the micrographs under crossed polarizers corresponding to the scattering patterns shown in Figure 4 for the solutions enclosed in a cell. The polarization directions are set vertically and horizontally. The micrograph at lower magnification (a) shows that the optically anisotropic domains are randomly oriented in space, while the micrograph at higher magnification (b) indicates that the domains have the cholesteric twisted structure as an internal structure. Since the shape of the domain is anisotropic, the small-angle scattering pattern is angularly dependent with respect to $\mu$. The cholesteric domain is again the structural origin of the rod of model $\mathrm{B}$ with $\omega_{0} \simeq 90^{\circ}$, giving rise to the X-type $H_{\mathrm{v}}$ pattern.

It should be noted that the cholesteric domain

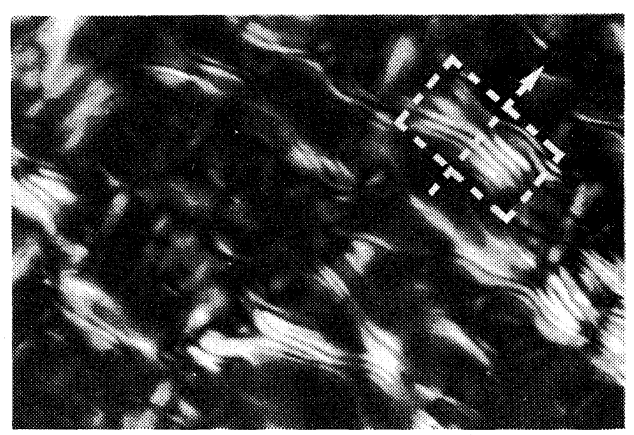

(a)

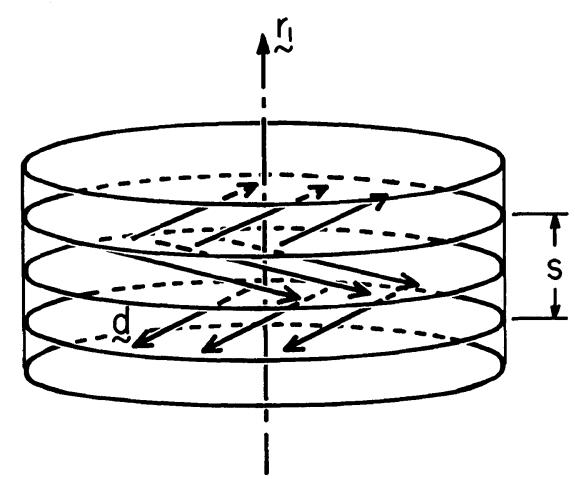

(b)

Figure 9. (a) Polarized-light micrograph corresponding approximately to the region giving rise to the scattering pattern shown in Figure 3 and (b) a schematic illustration of the cholesteric domain. The domain is a structural origin of the rod of model B with $\omega_{0} \simeq 90^{\circ}$ for the small-angle scattering. The polarization is set in vertical and horizontal direction.

generally grows with time after the solution at a given concentration is enclosed in the cell. Thus the small-angle scattering shifts toward smaller angles with time.

\section{EFFECT OF FORM-OPTICAL ROTATION ON THE ROD-LIKE SCATTERING}

When the rod-like structure is embedded in an optically active medium, the scattering should be affected by the optical rotatory power of the medium. This situation may be realized when the rod-like structure is embedded in a cholesteric liquid crystal medium having very high form-optical rotatory power. ${ }^{13,14}$ In this section, we shall discuss the effect of the form-optical rotation on rod-like 
PBLG-Dioxane $16.9 \mathrm{wt} \%$
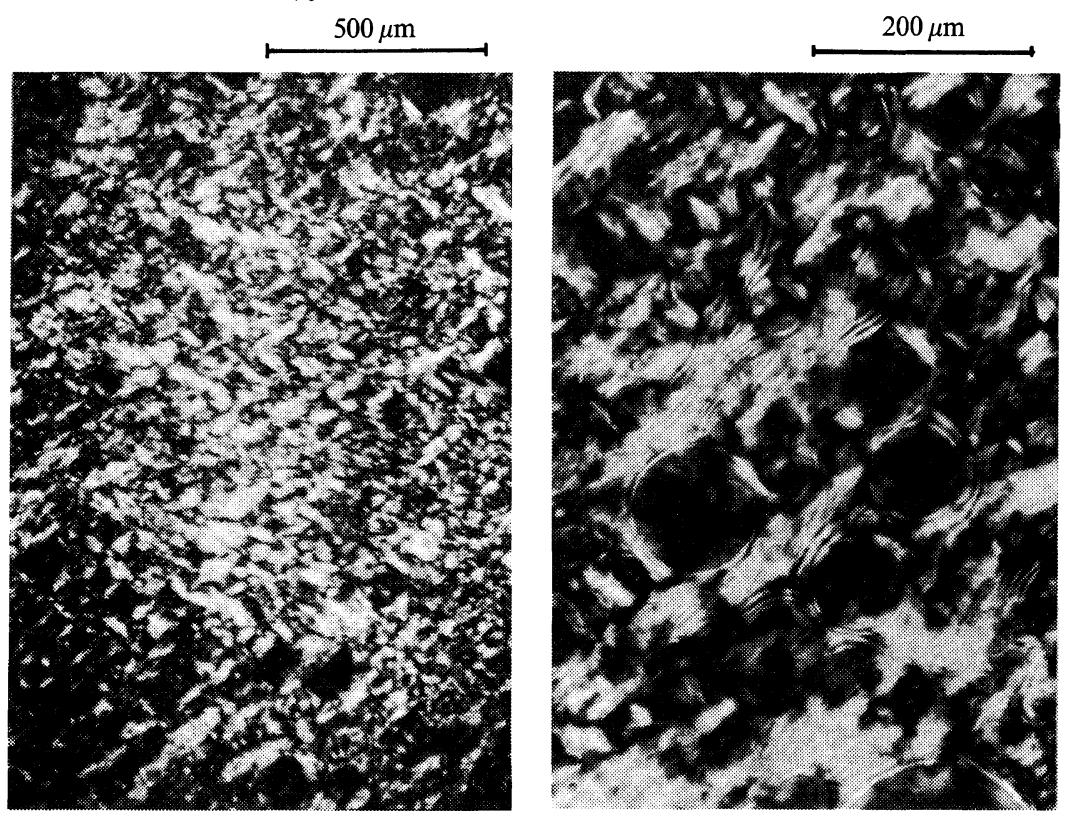

Figure 10. Polarized-light micrographs corresponding to the scattering patterns shown in Figure 4 . The polarization is set in vertical and horizontal directions. $17 \mathrm{wt} \%$ dioxane solution of PBLG-2 in a glass cell.

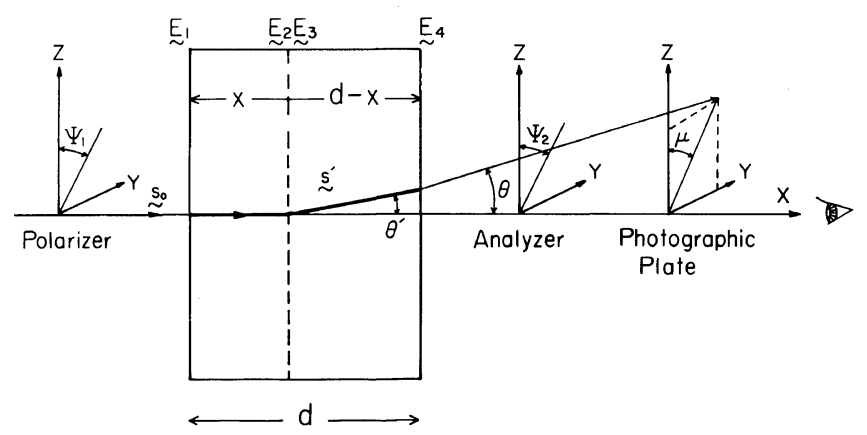

Figure 11. The definitions of the angles $\psi_{1}, \psi_{2}$, and $\mu$ which are taken to be positive when the observer toward whom the incident beam propagates can see a clockwise rotation of the respective vectors. $s_{0}$ and $s^{\prime}$ are the unit vectors along the popagation directions of the incident and scattered light.

scattering.

\section{Model}

We shall simplify our problem as follows, as in the model of Picot-Stein, ${ }^{18}$ who calculated the effect of the optical rotation on the spherulitic scattering. The anisotropic rod is assumed to be embedded in a uniform and optical active medium of thickness $d$ (see Figure 11). The medium is assumed to have a uniform form-optical rotatory power $K$, as described by de Vries, ${ }^{14}$

$$
K \equiv \mathrm{d} \psi / \mathrm{d} x=-\pi(\Delta n)^{2} P / 4 \lambda_{0}{ }^{2}
$$

for the case where $P \gg \lambda_{0}$ where $\Delta n$ is the birefringence of the untwisted material, $\lambda_{0}$ is the wavelength of light in vacuum and $P$ is the cholesteric pitch. The rods are considered to be randomly oriented in the optically active medium. 
In order to simplify the calculation, the rods are assumed to be randomly orinted in a plane $Y O Z$ normal to the propagating direction of the incident beam.

The plane of the polarization of the incident electric field $\boldsymbol{E}_{1}$ is rotated through the angle $\rho$ to result in $\boldsymbol{E}_{2}$ when it traverses the medium over a distance $x$, where in the rods under consideration are situated.

$$
\rho=K x
$$

We define $K$ as positive when the observer, toward whom the incident beam propagates, can see a clockwise rotation of the electric vector. Thus, it follows that,

$$
\begin{gathered}
\boldsymbol{E}_{1}=E_{0}\left(\sin \psi_{1} \hat{\boldsymbol{y}}+\cos \psi_{1} \hat{z}\right) \\
\boldsymbol{E}_{2}=E_{0}\left[\sin \left(\psi_{1}+\rho\right) \hat{\boldsymbol{y}}+\cos \left(\psi_{1}+\rho\right) \hat{\boldsymbol{z}}\right]
\end{gathered}
$$

where $\hat{\boldsymbol{y}}$ and $\hat{z}$ are unit vectors along the $y$ and $z$ axes. The scattered field $\boldsymbol{E}_{3}$ is then calculated from the state of polarization $E_{2}$ according to the scattering theory of the rod. The plane of polarization of the scattered field $\boldsymbol{E}_{3}$ is again rotated when it traverses the medium over a depth $(d-x)$ to result in $\boldsymbol{E}_{4}$. The fraction of $\boldsymbol{E}_{4}$ passing through the analyzer is then calculated.

The effect of the optical rotation on the $H_{\mathrm{v}}$ and $V_{\mathrm{v}}$ scattered intensity have been calculated by Stein-Picot and the final formula are given by eq 21 to 24 of reference 18 . Intensity terms like $A_{V_{\mathrm{v}}}^{2}, A_{H_{\mathrm{h}}}^{2}$, $A_{V_{\mathrm{h}}}^{2}$, and $A_{H_{\mathrm{v}}}^{2}$, and the cross-terms like $\left(A_{V_{\mathrm{v}}} A_{H_{\mathrm{h}}}+A_{V_{\mathrm{h}}} A_{H_{\mathrm{v}}}\right), A_{V_{\mathrm{v}}} A_{V_{\mathrm{h}}}, A_{V_{\mathrm{v}}} A_{H_{\mathrm{v}}}, A_{H_{\mathrm{h}}} A_{V_{\mathrm{h}}}$, and $A_{\mathrm{H}_{\mathrm{h}}} A_{\mathrm{H}_{\mathrm{v}}}$ should be calculated from the scattering theory of the rod.

\section{Rod-like Scattering}

We now consider scattering from the rod of model A which has infinitesimally thin lateral dimensions. When the plane containing the optical axes is confined in the $Y O Z$ plane (i.e., $\gamma=0$ in Figure 2(a)), the intensity and cross-terms discussed above are given by

$$
\begin{aligned}
A_{H_{\mathrm{v}}}^{2} & =A_{V_{\mathrm{h}}}^{2} \\
& =\frac{1}{2} \int_{0}^{\pi / 2}\left(1-\cos 4 \zeta_{0}\right) \cos 4 t j_{0}^{2}(w \cos t) \mathrm{d} t
\end{aligned}
$$

$$
\begin{aligned}
A_{H_{\mathrm{h}}}^{2}= & \int_{0}^{\pi / 2}\left[\frac{1}{2} \cos 4 \zeta_{0} \cos 4 t\right. \\
& -4\left(p+\frac{1}{2}\right) \cos 2 \zeta_{0} \cos 2 t \\
& \left.+4\left(\frac{3}{8}+p+p^{2}\right)\right] \times j_{0}^{2}(w \cos t) \mathrm{d} t
\end{aligned}
$$

$$
\begin{aligned}
A_{V_{\mathrm{v}}}^{2}= & \int_{0}^{\pi / 2}\left[\frac{1}{2} \cos 4 \zeta_{0} \cos 4 t\right. \\
& +4\left(p+\frac{1}{2}\right) \cos 2 \zeta_{0} \cos 2 t \\
& \left.+4\left(\frac{3}{8}+p+p^{2}\right)\right] \times j_{0}^{2}(w \cos t) \mathrm{d} t
\end{aligned}
$$

$$
\begin{aligned}
A_{V_{\mathrm{v}}} A_{H_{\mathrm{h}}} & +A_{V_{\mathrm{h}}} A_{H_{\mathrm{v}}} \\
& =2 A_{H_{\mathrm{v}}}^{2}+4\left(p+p^{2}\right) \int_{0}^{\pi / 2} j_{0}^{2}[w \cos t] \mathrm{d} t
\end{aligned}
$$

$$
\begin{aligned}
A_{H_{\mathrm{v}}} A_{H_{\mathrm{h}}}= & A_{V_{\mathrm{h}}} A_{H_{\mathrm{h}}} \\
= & \int_{0}^{\pi / 2}\left[-\frac{1}{2} \sin 4 \zeta_{0} \cos 4 t\right. \\
& \left.+(1+2 p) \sin 2 \zeta_{0} \cos 2 t\right] j_{0}^{2}[w \cos t] \mathrm{d} t
\end{aligned}
$$

$$
\begin{aligned}
A_{V_{\mathrm{v}}} A_{H_{\mathrm{v}}}= & A_{V_{\mathrm{v}}} A_{V_{\mathrm{h}}} \\
= & \int_{0}^{\pi / 2}\left[\frac{1}{2} \sin 4 \zeta_{0} \cos 4 t\right. \\
& \left.+(1+2 p) \sin 2 \zeta_{0} \cos 2 t\right] j_{0}^{2}[w \cos t] \mathrm{d} t
\end{aligned}
$$

where

$$
\begin{aligned}
W & =\pi(L / \lambda) \sin \theta^{\prime} \\
p & =\left(\alpha_{\perp}-\alpha_{\mathrm{s}}\right) /\left(\alpha_{\|}-\alpha_{\perp}\right) \\
\zeta_{0} & =\mu+\omega_{0}
\end{aligned}
$$

$L$ is the length of the rod, $\theta^{\prime}$ is the scattering angle in the medium, $\alpha_{\mathrm{s}}$ is the polarizability of the medium surrounding the rod $\left(\alpha_{\mathrm{s}}\right.$ being assumed to be a scalar quantity), $j_{0}$ is the zeroth-order spherical Bessel function of the first kind. These calculations are performed for the case where the rods having $+\omega_{0}$ and $-\omega_{0}$ are equally probable. In such a case, one 
should replace, respectively, $\cos n \zeta_{0}$ and $\sin n \zeta_{0}$ by $\cos n \zeta_{0}=\cos n \mu \cos n \omega_{0}$, $\sin n \zeta_{0}=\sin n \mu \cos n \omega_{0}$

$(n=4$ or 2$)$

$\omega_{0}=50^{\circ}, p=-\sqrt{3}$

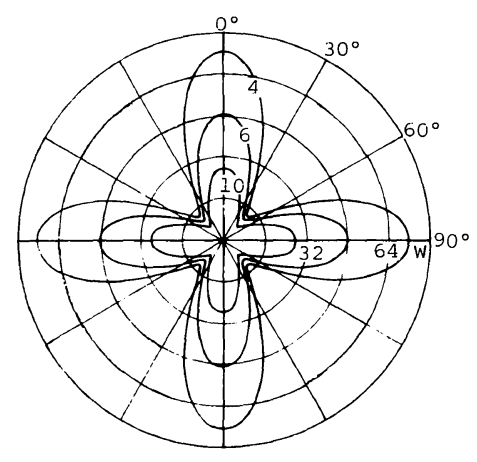

(a) $\mathrm{Kd}=0^{\circ}$

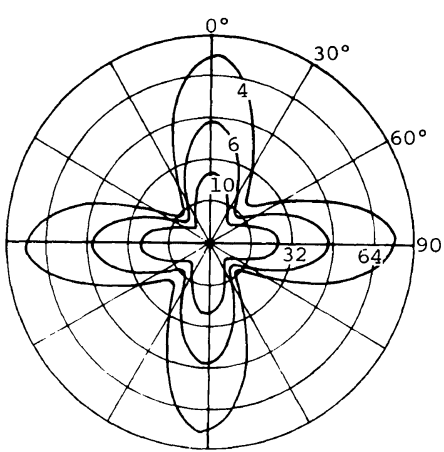

(b) $\quad K d=30^{\circ}$

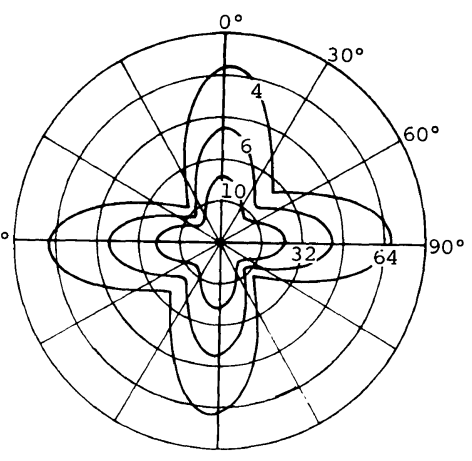

(c) $\quad K d=45^{\circ}$

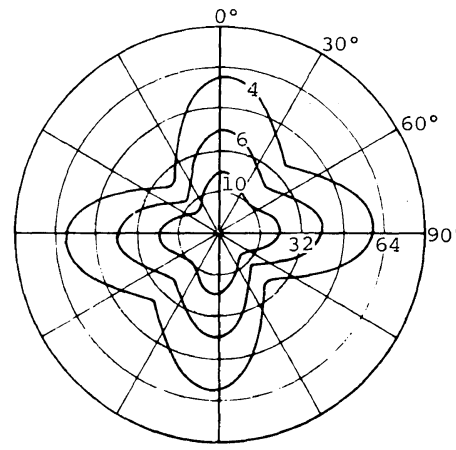

(d)

$\mathrm{Kd}=60^{\circ}$

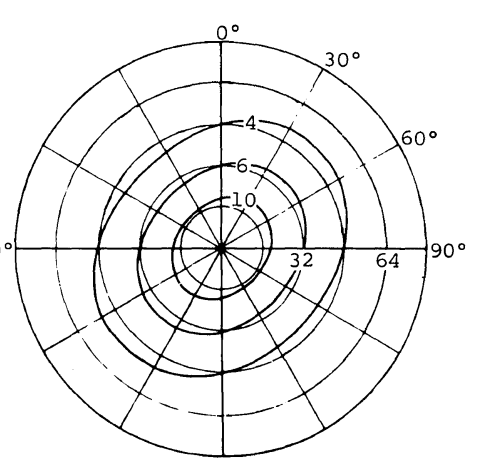

(e) $\mathrm{Kd}=90^{\circ}$

Figure 12. Effect of the form-optical rotation on rod-like scattering showing +-type $H_{\mathrm{V}}$ pattern. $\omega_{0}=50^{\circ}$ and $p=-1 / 3$.

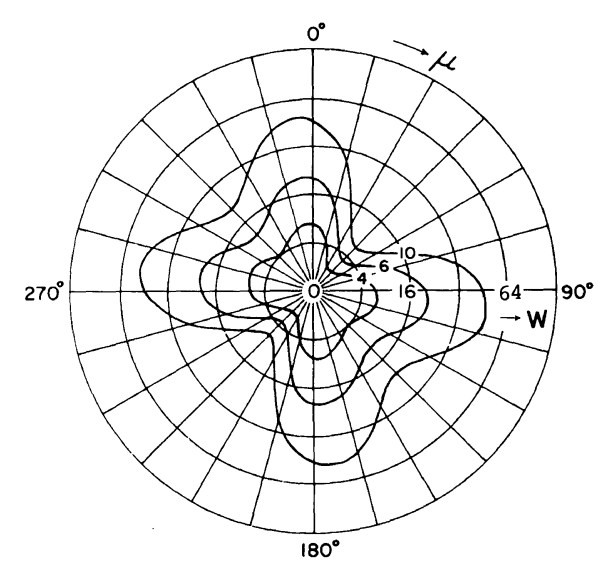

(a)

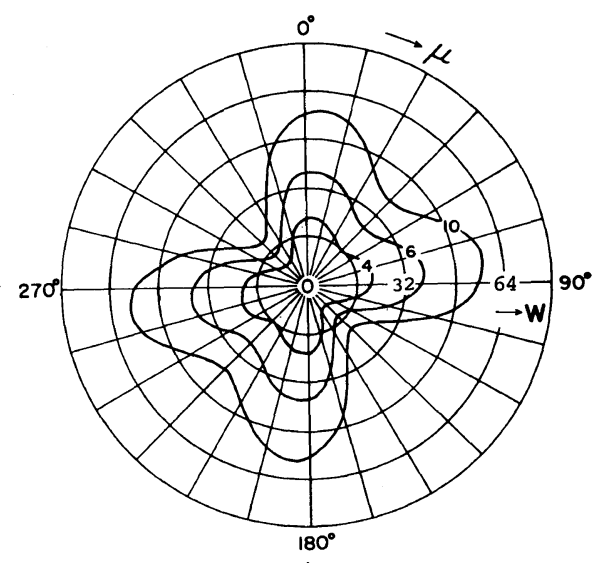

(b)

Figure 13. Effect of the sign of the optical rotatory power $K$ on the distortion of + -type $H_{\mathrm{v}}$ pattern, (a) $K d=-60$ and (b) $K d=60^{\circ}$ for $\omega_{0}=50^{\circ}$ and $p=-1 / 3$. 


\section{Results of Numerical Calculations}

Figures 12 to 16 show the results of numerical calculations on the effects of form-optical rotation on the rod-like scattering from the rod of model A. The effect on scattering from the rod of the model B should be identical to that from the rod of model A with $\omega_{0}=0^{\circ}$.

Figure 12 shows the effect of the form-optical rotation on the +-type $H_{\mathrm{v}}$ rod-like scattering for $\omega_{0}=50^{\circ}$ and $p=-1 / 3$. When $K d=0$, the medium is isotropic and therefore there should be no effect made by a form-optical rotation. Consequently, the scattering pattern has a+-type intensity distribution. As the effect of rotation increases, the pattern is distorted, and the four-fold symmetry in the $\mu$-dependence becomes loose, resulting in the two-fold symmetry around the azimuthal angle of $\mu=+45^{\circ}$ and $225^{\circ}$ in the system having a positive rotatory power $(K>0)$ and $p<0$.

When the rotatory power is negative, i.e., $K<0$, the $H_{\mathrm{v}}$ pattern is distorted in an opposite direction as shown in Figure 13, where the patterns (a) and (b) are obtained for $K d=-60^{\circ}$ and $K d=+60^{\circ}$, respectively, for $H_{\mathrm{v}}$ polarizations, $\omega_{0}=50^{\circ}$ and $p=-1 / 3$, i.e.,

$$
I_{H_{\mathrm{v}}}(-K, p ; \mu)=I_{H_{\mathrm{v}}}\left(K, p ; \mu+90^{\circ}\right)
$$

Pattern (a) is obtained with a rotation of the pattern (b) by $90^{\circ}$. Thus, the manner of distortion may be a measure of the sign of the form-optical

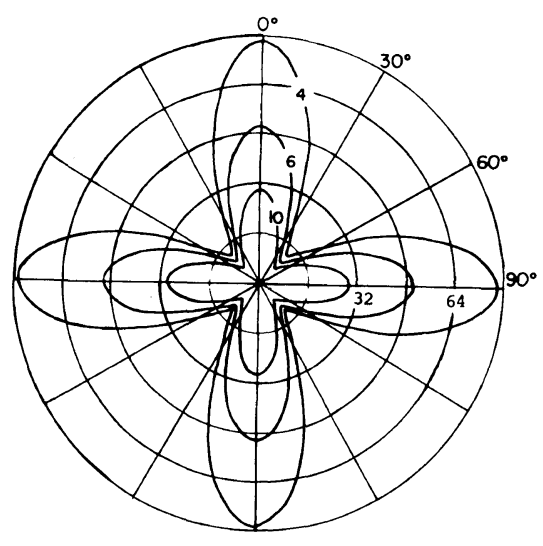

(a) $\mathrm{Kd}=0^{\circ}$

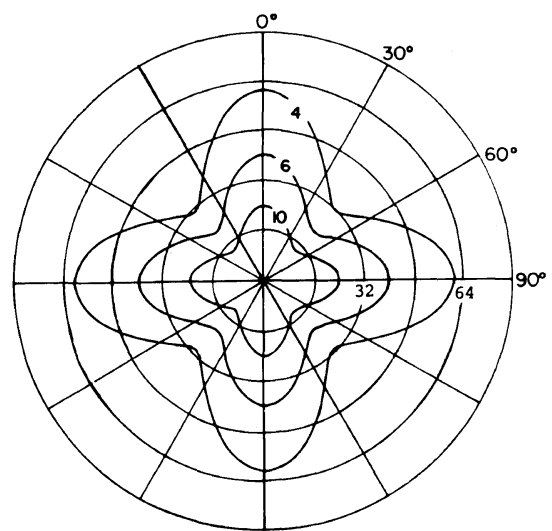

(c) $\mathrm{Kd}=60^{\circ}$

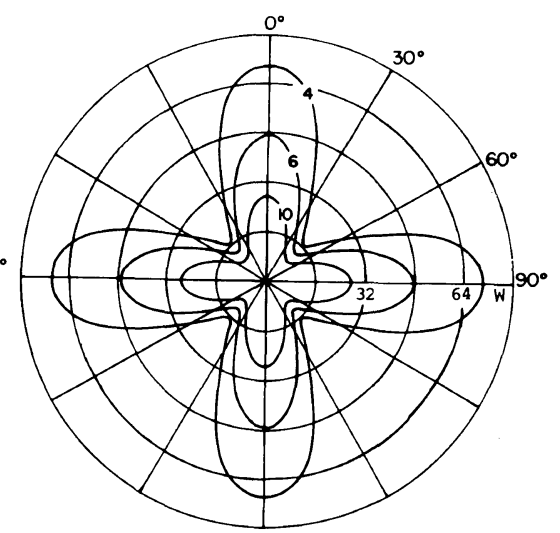

(b) $\mathrm{Kd}=30^{\circ}$

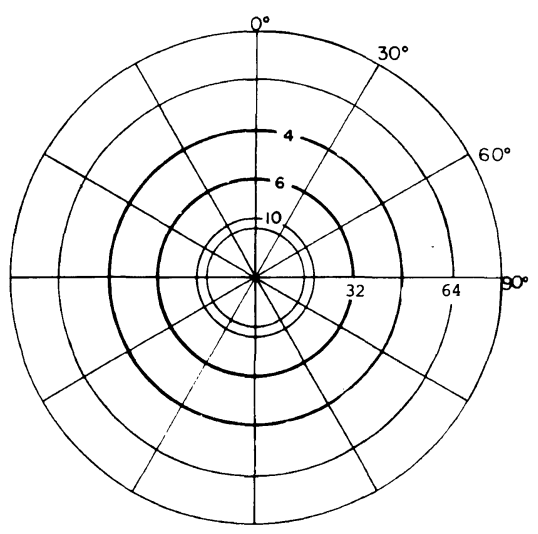

(d) $\mathrm{Kd}=90^{\circ}$

Figure 14. Effect of the form-optical rotation on rod-like scattering showing +-type $H_{\mathrm{V}}$ pattern. $\omega_{0}=45^{\circ}$ and $p=-1 / 3$. 
rotation and therefore the sense of the cholesteric twisting, provided that the sign of $p$ is known. It should be noted that the distortion of the pattern depends also on the sign of $p$, since the observed $V_{\mathrm{H}}$ or $H_{\mathrm{V}}$ scattering depends on the $V_{\mathrm{V}}$ and $H_{\mathrm{H}}$ components of the scattered light as well as the true depolarized component resulting from the optical rotation.

In a particular case in which $\omega_{0}=45^{\circ}$, the distortion of the $H_{\mathrm{v}}$ pattern cannot be observed as shown in Figure $14(p=-1 / 3)$. That is, with an increase in the effect of the optical rotation, the $\mu$ dependence of the pattern decreases, resulting in the circularly symmetric pattern shown in Figure 14(d) but the four-fold symmetry of the pattern is conserved.

Figure 15 shows the effect of optical rotation on the X-type $H_{\mathrm{v}}$ patterns ( $\left.p=-1 / 3\right)$. Again, the effect distorts the four-fold symmetry of the scattering patterns with respect to $\mu$. The greater the effect, the larger the distortion. For given values of $K$ and $p$, the manner of the distortion depends on $\omega_{0}$. Also for given values of $\omega_{0}$ and $p$, the distortion of the pattern is reversed by changing the sign of $K$, indicating that eq 13 is valid for $\omega_{0}=0^{\circ}$ and $90^{\circ}$ as well. In fact, it can be shown analytically that eq 13 is valid for all values of $\omega_{0}$ at small scattering angles where $\Omega_{3}^{\prime} \simeq \Omega_{4}^{\prime}, \Omega_{6}^{\prime} \simeq \Omega_{7}^{\prime}$, and $\Omega_{8}^{\prime} \simeq \Omega_{9}^{\prime}$ in eq 24 of Stein-Picot. ${ }^{18}$

Figure 16 shows the effect of optical rotation on the $V_{\mathrm{v}}$ scattering patterns from the rod having $\omega_{0}=50^{\circ}$ and $p=-1 / 3$. This effect diminishes the $\mu$ dependence of the $V_{\mathrm{V}}$ scattering but does not distort the symmetry of the pattern with $\mu$. A similar effect should be expected on the $H_{\mathrm{H}}$ patterns.

\section{Comparisons with Experimental Results}

The distortion of the depolarized small-angle light scattering patterns which are observed for the concentrated solutions of PBLG specimens (as shwn in Figures 3 and 4) may be attributed primarily to the effect of the form-optical rotation. As shown in Figure 4 the distortion of the $H_{\mathrm{v}}$ scattering is opposite to that of the $V_{\mathrm{H}}$ scattering, thus further confirming that the distortion originates from the optical activity. In fact one can analytically varify that the $H_{\mathrm{V}}\left(V_{\mathrm{H}}\right)$ pattern is obtained with a rotation of $V_{\mathrm{H}}\left(H_{\mathrm{V}}\right)$ pattern by $90^{\circ}$ around the incident beam

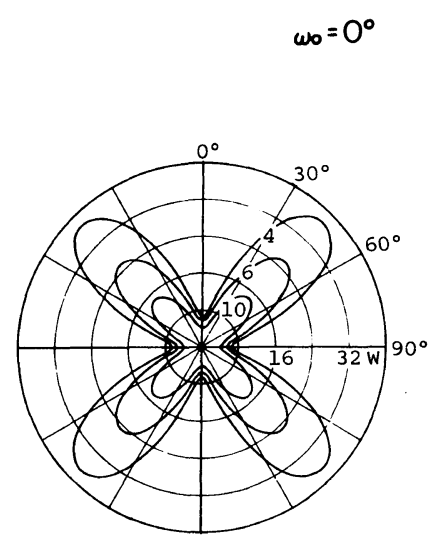

$\omega_{0}=90^{\circ}$

$\mathrm{Kd}=0^{\circ}$
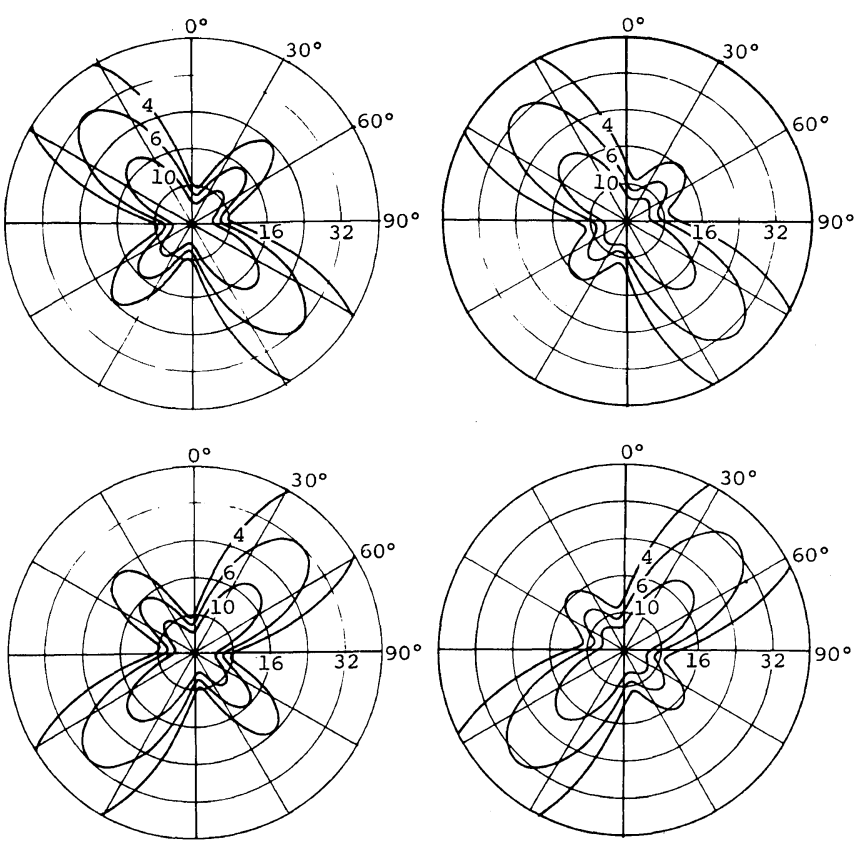

$\mathrm{Kd}=30^{\circ}$

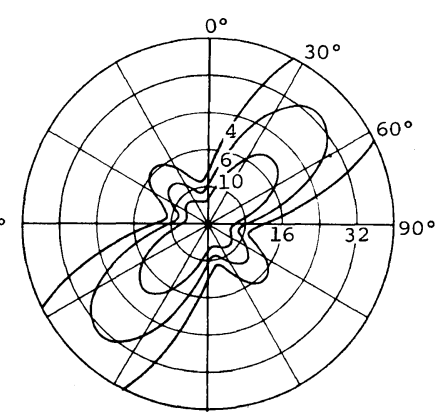

$K d=45^{\circ}$

Figure 15. Effect of the form-optical rotation on X-type $H_{\mathrm{V}}$ patterns for $\omega_{0}=0^{\circ}$ and $90^{\circ} \cdot p=-1 / 3$. 


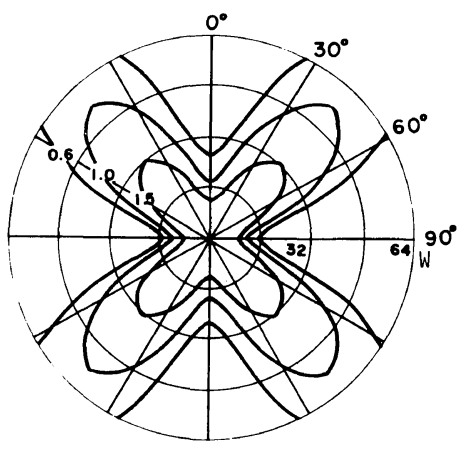

$K d=0^{\circ}$

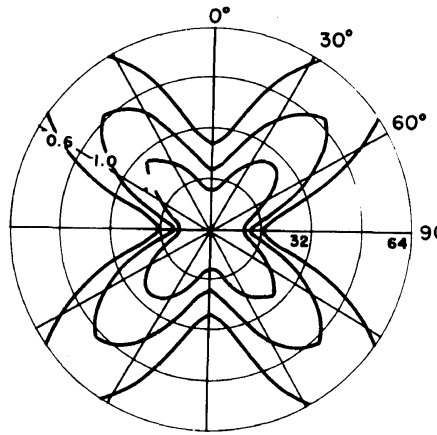

$\mathrm{Kd}=30^{\circ}$

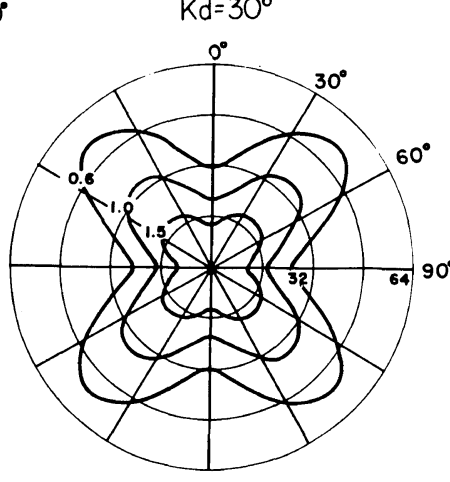

$\mathrm{Kd}=60^{\circ}$

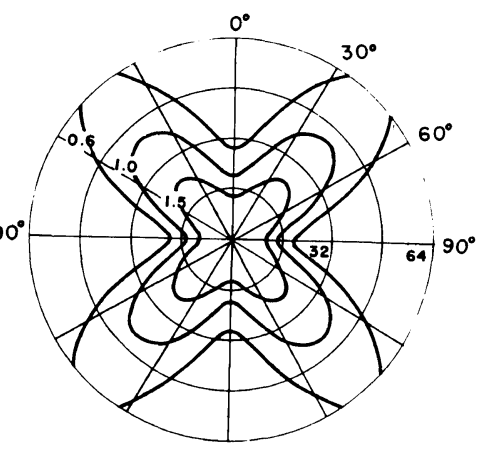

$K d=45^{\circ}$

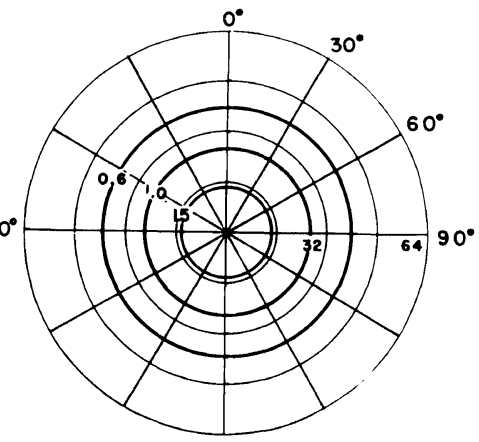

$K d=90^{\circ}$

Figure 16. Effect of the form-optical rotation on $V_{\mathrm{V}}$ scattering patterns. $\omega_{0}=50^{\circ}$ and $p=-1 / 3$.

axis, i.e.,

$$
I_{H_{\mathbf{v}}}\left(K, p, \omega_{0} ; \mu\right)=I_{V_{\mathbf{h}}}\left(K, p, \omega_{0} ; \mu+90^{\circ}\right)
$$

The distortion of the depolarized pattern is sometimes observed for the +-type pattern in the solid films shown in Figures 7 and 17. However, the origin of the distortion shown in Figure 7 is not so clear as that of the scattering from the systems of the cholesteric liquid crystals discussed above, since the distortion of $H_{\mathrm{v}}$ pattern is neither exactly opposite nor identical to that of the $V_{\mathrm{H}}$ pattern. In many cases, as shown in Figure $17 H_{\mathrm{V}}$ and $V_{\mathrm{H}}$ patterns are distorted in nearly the same manner. In such cases it is clear that this distortion should primarily arise from macroscopic orientation of the cholesteric domains rather than result from the optical activity.

The effect of the optical rotation on the "ring" scattering, i.e., the scattering maximum arising from the cholesteric twisting will be described in detail in a subsequent paper. ${ }^{15}$

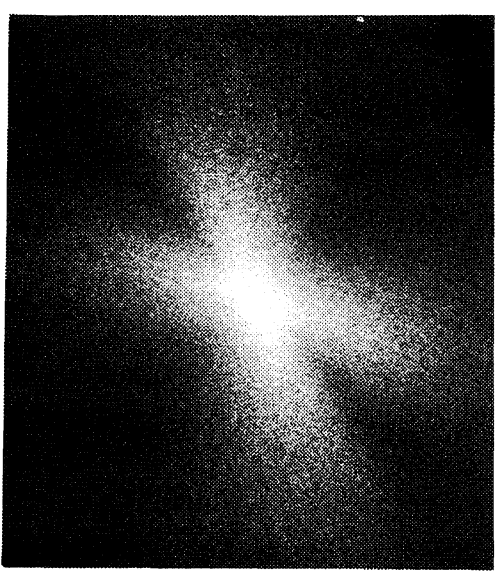

Figure 17. Distorted +-type $H_{\mathrm{v}}$ patterns from a film cast from $2 \mathrm{wt} \%$ TCE solution.

Acknowledgment. The authors are deeply indebted to Dr. T. Hayashi and professor A. Nakajima, Kyoto University for kindly preparing the poly $(\gamma-$ benzyl L-glutamate) samples. 


\section{REFERENCES}

1. R. S. Stein and P. R. Wilson, J. Appl. Phys., 33, 1914 (1962).

2. R. S. Stein and M. B. Rhodes, J. Appl. Phys., 31, 1873 (1960).

3. R. S. Stein, in "Structure and Properties of Polymer Films," R. W. Lenz and R. S. Stein, Ed., Plenum Press, N.Y., 1973.

4. M. B. Rhodes and R. S. Stein, J. Polym. Sci., A-2, 7, 1539 (1969).

5. T. Hashimoto, Y. Murakami, N. Hayashi, and H. Kawai, Polym. J., 6, 132 (1974).

6. M. Matsuo, S. Nomura, T. Hashimoto, and $\mathrm{H}$. Kawai, Polym. J., 6, 151 (1974).

7. T. Hashimoto, Y. Murakami, and H. Kawai, $J$. Polym. Sci.,Polym. Phys. Ed., 13, 1613 (1975).

8. T. Hashimoto, K. Yamaguchi, and H. Kawai, Polym.
$J ., 9,405$ (1977).

9. M. Moritani, N. Hayashi, A. Utsuo, and H. Kawai, Polym. J., 2, 74 (1971).

10. N. Hayashi and H. Kawai, Polym. J., 3, 140 (1972).

11. Y. Murakami, N. Hayashi, T. Hashimoto, and H. Kawai, Polym. J., 4, 452 (1973).

12. J. Mathieu, J. Bull. Soc. Trans. Minerol., 34, 13 (1911).

13. C. Robinson, Molecular Cryst., 1, 467 (1966).

14. Hl. de Vries, Acta Cryst., 4, 219 (1951).

15. T. Hashimoto, S. Ebisu, N. Inaba, and H. Kawai, to be submitted to J. Polym. Sci., Polym. Phys. Ed.

16. T. Hashimoto, S. Ebisu, and H. Kawai, J. Polym. Sci., Polym. Lett. Ed., 18, 569 (1980).

17. S. Ishikawa and T. Kurita, Biopolymers, 2, 381 (1964).

18. C. Picot and R. S. Stein, J. Polym. Sci., A-2, 8, 1491 (1970). 